\title{
Gérard Grisey : première section de Partiels (1975)
}

\section{François-Xavier Féron}

\section{(2) OpenEdition}

\section{Journals}

Édition électronique

URL : http://journals.openedition.org/genesis/352

DOI : 10.4000/genesis.352

ISSN : 2268-1590

\section{Éditeur :}

Presses universitaires de Paris Sorbonne (PUPS), Société internationale de génétique artistique littéraire et scientifique (SIGALES)

\section{Édition imprimée}

Date de publication : 10 octobre 2010

Pagination : 77-97

ISBN : 978-2-84050-711-6

ISSN : 1167-5101

\section{Référence électronique}

François-Xavier Féron, « Gérard Grisey : première section de Partiels (1975) », Genesis [En ligne], 31 | 2010, mis en ligne le 21 septembre 2012, consulté le 30 avril 2019. URL : http:// journals.openedition.org/genesis/352; DOI : 10.4000/genesis.352 


\section{Gérard Grisey : première section de Partiels (1975)}

François-Xavier Féron

$P$ RTIELS ${ }^{1}$ A ÉTÉ COMPOSÉ EN 1975 par Gérard Grisey (1946-1998) et créé le 4 mars 1976 à Paris par l'ensemble l'Itinéraire sous la direction de Boris de Vinogradov. Cette œuvre emblématique de la musique «spectrale » puise une partie de son inspiration dans les connaissances acoustiques sur le spectre sonore. Aborder la genèse de Partiels ne peut se faire sans évoquer Périodes (1974) pour sept instrumentistes car, dans la dernière section de cette partition, Grisey fait jouer par l'ensemble des instruments les composantes d'un spectre harmonique, procédé qui, au regard de son originalité, de ses répercussions sonores, mais aussi de son caractère suspensif, appelait un développement :

Lorsque j'ai composé Périodes je me suis aperçu que la fin n'était pas une fin, qu'il fallait une suite, et j'ai imaginé Partiels qui a été composé immédiatement après. Et comme Périodes commençait par un alto seul, évidemment immédiatement est venue l'idée de faire une sorte de prologue pour tout ce cycle, et peu à peu est né le concept d'une grande pièce $^{2}$.

Les six partitions (Prologue, Périodes, Partiels, Modulations, Transitoires, Épilogue) constituant Les espaces acoustiques (cycle d'une durée totale avoisinant les quatre-vingt-dix minutes) sont composées entre 1974 et 1985. Les titres révèlent l'emprise de l'acoustique dans le sens le plus large du terme, c'est-à-dire «la Science générale des sons perçus et intégrés par l'homme ${ }^{3}$ », sur les orientations musicales du compositeur. Le titre Partiels «s'entend comme moment d'un ouvrage plus vaste, mais aussi dans le sens acoustique de composantes du son 4 ». Bien que cette œuvre ait fait l'objet de nombreuses analyses musicales à la lecture desquelles nous renvoyons ${ }^{5}$, aucune d'entre elles ne l'aborde sous l'angle génétique. Le dossier ici présenté se focalise sur cette sorte de manifeste de l'esthétique «spectrale» que représente la première section de Partiels. Sont reproduits, avec l'aimable autorisation de la fondation Paul Sacher à Bâlé 6 , quelques manuscrits de la collection

1. Partiels est écrit pour un ensemble de chambre de dix-huit musiciens (la partition peut être exécutée avec un seul corniste et un seul percussionniste moyennant quelques adaptations que Grisey précise dans la notice). Voici l'effectif instrumental accompagné des abréviations telles qu'elles apparaissent sur la partition (Ricordi 132423, 1976) : 2 flûtes jouant flûte piccolo $[\mathrm{Fl}$ pic] et flûtes alto $[\mathrm{Fl}] ; 1$ hautbois $[\mathrm{Ob}]$ prenant aussi le cor anglais [C ing] ; 2 clarinettes [Cl] en si $b$ : le premier jouant aussi la clarinette en $m i b$ et le second la clarinette en $l a ; 1$ clarinette basse $[\mathrm{Cl} \mathrm{B}]$ jouant aussi la clarinette contrebasse $[\mathrm{Cl} \mathrm{Cb}] ; 2$ cors [Cor] ; 1 trombone complet [Trbn] muni des sourdines Plunger, Bol, Robinson, Wawa et Velvet ; 1 accordéon avec basse chromatique [Fisarm]

2 percussions [Perc] ; 2 violons [Vn] ; 2 altos [Vle] ; 1 violoncelle [Vc] ; 1 contrebasse $[\mathrm{Cb}]$.

2. Gérard Grisey, entretien radiophonique avec Marc Texier en 1993, cité par Jérôme Baillet, Gérard Grisey. Fondements d'une écriture, Paris, L'Itinéraire/L'Harmattan, 2000, p. 70. La « sorte de prologue » sera composée peu après : Prologue pour alto solo (1976).

3. Émile Leipp, Acoustique et musique, Paris, Masson, 1971, 1996 (quatrième édition), p. 1.

4. Note de programme pour Partiels, dans Gérard Grisey, Écrits ou l' invention de la musique spectrale, édition établie par Guy Lelong avec la collaboration d'Anne-Marie Réby, Paris, Éditions MF, 2008, p. 137.

5. Voir Peter Niklas Wilson, "Vers une écologie des sons", Partiels de Gérard Grisey et l'esthétique du groupe de l'Itinéraire », article paru originellement en langue allemande (Melos, $\mathrm{n}^{\circ} 2,1988$, p. 33-55) puis traduit en français par M. Kaltenecker (Entretemps, ${ }^{\circ}{ }^{8}$, dossier « Grisey Murail », p. 55-81 ou Analyse musicale, $\mathrm{n}^{\circ}$ 36, 2000, p. 36-52) ; Jérôme Baillet, «Partiels de Gérard Grisey », L'Éducation musicale, supplément aux n 473/474, 2000, p. 47-56; Yves Krier, « Partiels de Gérard Grisey, manifestation d'une nouvelle esthétique », Musurgia, VII/3-4, 2000, p. 145-172 ; analyse de Thierry Alla : <http://webetab.ac-bordeaux.fr/ Pedagogie/Musique/grisey.html> ; analyse de Philippe Lalitte : <http:// musique.ac-dijon.fr/bac2001/grisey/>.

6. Je tiens à remercier très chaleureusement Robert Piencikowski, Henrike Hoffmann et Johanna Blask de la fondation Paul Sacher pour l'accueil et la disponibilité dont ils ont fait preuve à mon égard. 
Gérard Grisey permettant d'entrevoir comment le compositeur a imaginé et structuré les processus de transformation graduelle affectant d'une part le motif rythmique joué à la contrebasse et d'autre part le spectre reconstitué.

Pour appréhender de tels documents, il est nécessaire d'expliciter quelques notions d'acoustique (spectre harmonique/inharmonique, partiel, zone formantique, transitoires d'attaque et d'extinction, son différentiel...). Nous tenterons d'ailleurs de comprendre comment Grisey a lui-même assimilé ces notions. Nous décrirons ensuite le procédé de «synthèse instrumentale » grâce auquel il restitue un spectre et dont le rôle, dans la genèse de Partiels, s'avère primordial. Enfin, nous présenterons un groupe d'avant-textes (notes manuscrites, plans synoptiques réalisés à la main sur un assemblage de papier musique et papier petits carreaux...) illustrant d'une part la genèse de l'idée centrale de cette section (métamorphose du spectre), d'autre part la méthodologie et logique compositionnelle mises en œuvre par le compositeur.

\section{L'acoustique mise en musique : de la synthèse additive à la « synthèse instrumentale »}

Un son complexe ayant une hauteur parfaitement définie (tel que celui produit par la plupart des instruments de musique) est composé d'une multitude de composantes spectrales appelées partiels harmoniques (ou tout simplement harmoniques) car leurs fréquences sont en rapport harmonique avec la fréquence fondamentale $: \mathrm{f}_{\mathrm{n}}=\mathrm{n} \times \mathrm{f}_{0}$ avec $\mathrm{f}_{\mathrm{n}}$, la fréquence de la énième composante spectrale et $\mathrm{f}_{0}$, la fréquence de la fondamentale 7 . La hauteur perçue est étroitement liée (mais pas seulement) à cette fréquence fondamentale : plus celle-ci est petite, plus le son est grave. Le timbre du son est en partie conditionné par la répartition spectrale, c'est-à-dire le nombre et l'amplitude des composantes spectrales.

Un son composé d'une seule fréquence est qualifié de pur : son timbre est extrêmement pauvre8. Un son musical présente en revanche une structure spectrale beaucoup plus complexe, ce qui rend son timbre plus riche. La figure 1 est un sonagramme d'un $m i_{1}{ }^{9}$ (fondamentale $=41,2 \mathrm{~Hz}$ ) joué au trombone : une telle représentation (fréquence en ordonnée, temps en abscisse, intensité selon la noirceur des traits) permet d'apprécier l'évolution temporelle des composantes spectrales et de rendre ainsi compte de la structure interne du son. L'équidistance des raies dans ce cas précis témoigne de l'harmonicité du spectre mais il arrive que les fréquences ne soient plus des multiples entiers de la fondamentale, comme dans le cas d'un son de cloche par exemple : on parle alors de partiels inharmoniques (ou tout simplement de partiels) et de spectre inharmonique. Le titre choisi par Grisey n'est pas métaphorique ou anecdotique mais se réfère justement à l'intrusion de telles composantes dans le spectre qu'il restitue musicalement. Enfin, le bruit est un signal extrêmement riche du point de vue spectral. Les composantes sont tellement rapprochées qu'elles forment des bandes fréquentielles : la partie verticale noircie au début du sonagramme traduit justement la présence de bruit qui, en l'occurrence, correspond au souffle de l'interprète lorsque celui-ci entonne la note.

$\mathrm{Si}$ un son musical peut être analysé comme une somme de composantes spectrales, il est a priori envisageable de construire un son complexe à partir de sons purs dont on contrôle chaque paramètre. Cette technique de construction par addition de sons primaires (le plus souvent purs) est communément appelée synthèse additive. Explorée depuis des siècles par les facteurs d'orgue qui, grâce aux différents trous percés sur un registre, font sonner plusieurs tuyaux en même temps dont « l'ensemble donne une note unique, mais de timbre particulier $10 »$, elle a favorisé le développement à la fin du XIXe siècle de la lutherie électromécanique, puis au $\mathrm{xx}^{\mathrm{e}}$ siècle, des musiques électroniques, de la synthèse

7. Selon cette nomenclature, il apparaît que $\mathrm{f}_{0}$ est confondue avec $\mathrm{f}_{1}$, c'est-à-dire que l'harmonique 1 correspond à la fondamentale.

8. La tonalité du téléphone correspond généralement à un son pur (moyennant quelques distorsions) de $440 \mathrm{~Hz}$.

9. Les numéros associés aux notes désignent conventionnellement les octaves selon la nomenclature française. Le do le plus grave sur un piano correspond à $d o_{0}(32,7 \mathrm{~Hz})$, le plus aigu (la dernière touche du clavier) à $d o_{7}(4186 \mathrm{~Hz})$. Les instruments de l'orchestre s'accordent généralement à partir du $l a_{3}(440 \mathrm{~Hz})$.

10. Émile Leipp, Acoustique et musique, op. cit., p. 262. 


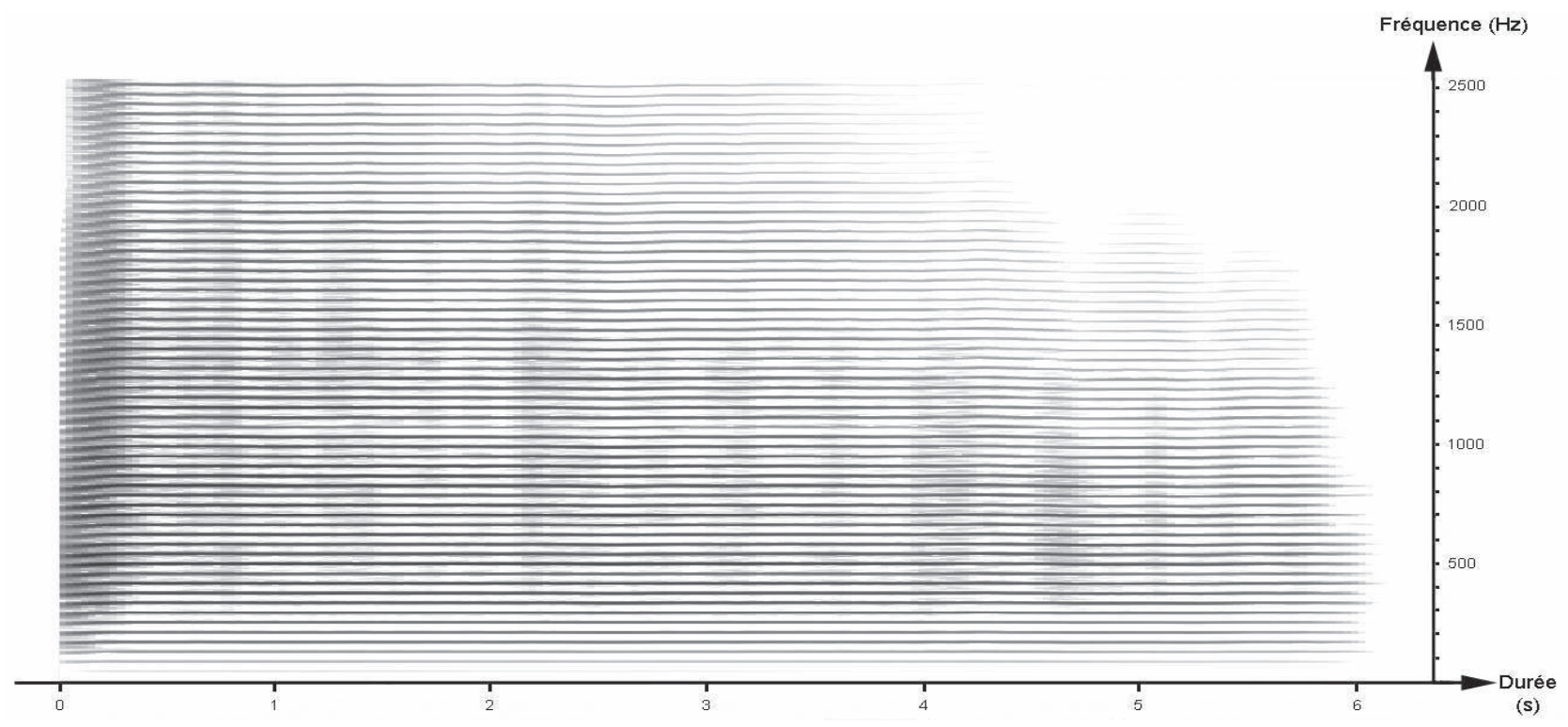

Fig. 1 : Sonagramme d'un $m i_{0}\left(\mathrm{f}_{0}=41,2 \mathrm{~Hz}\right)$ de trombone basse réalisé en 2010 avec le logiciel Audiosculpt à partir d'un échantillon sonore enregistré à l'Ircam. Chaque raie horizontale représente l'évolution au cours du temps d'une composante spectrale du son (la fenêtre fréquentielle est limitée à $2500 \mathrm{~Hz}$ de sorte à pouvoir apprécier l'équidistance entre chaque raie).

La bande verticale sombre au début du sonagramme rend compte du bruit provoqué par le souffle de l'interprète lors de l'attaque du son

sonore par ordinateur... La « synthèse instrumentale11 » imaginée par Grisey dès 1973 est calquée sur le principe de synthèse additive, mais au lieu de travailler avec des sons primaires, il emploie des sons instrumentaux. Comment ce compositeur sans formation scientifique préalable a-til pu imaginer un tel procédé ? Comment parvient-il à le transcrire musicalement, sachant qu'un instrument de musique n'est ni un simple tuyau d'orgue, ni un appareillage physique dont on peut contrôler précisément la fréquence d'émission?

Bien que l'impact de l'acoustique sur l'ensemble de son œuvre soit très souvent mentionné dans ses écrits ou dans les articles qui lui sont consacrés, nous n'avons que très peu de renseignements quant à l'apprentissage de ce savoir. Grisey suit des cours de musique électroacoustique avec Jean-Étienne Marie en 1969 : il aurait pu, dans ce contexte, aborder quelques notions scientifiques. Il étudie aussi l'acoustique musicale à la faculté des sciences de Paris auprès d'Émile Leipp en 1974-197512, mais des œuvres comme Dérives (19731974) pour deux groupes d'orchestre et Périodes (1974) pour sept instrumentistes (toutes deux composées durant son séjour à la villa Médicis à Rome) mettaient déjà en jeu des procédés compositionnels s'inspirant de l'acoustique. En examinant à la fondation Paul Sacher les ouvrages que Grisey possédait, nous avons pu constater que le compositeur s'est surtout formé dans un premier temps de manière autodidacte, et cela dès 1972-1973, comme en attestent la signature et la datation des pages de garde de ses exemplaires d'Acoustique et musique d'Émile Leipp et de Vues nouvelles sur le monde des sons de Fritz Winckel13 (voir fig. 2). Ces deux ouvrages, d'une pédagogie exemplaire, sont les premiers du genre en langue française et s'adressent à tous ceux et celles qui

11. Bien que ce procédé soit exploré dès 1973, Grisey semble employer ce terme pour la première fois dans un article dactylographié daté de février 1979 : « À propos de la synthèse instrumentale », dans Gérard Grisey, Écrits, op. cit., p. 35-37.

12. «Chronologie 1946-1998 », dans Gérard Grisey, Écrits, op. cit., p. 331 .

13. Fritz Winckel, Vues nouvelles sur le monde des sons, Paris, Dunod, 1960 . 
désirent posséder des connaissances fondamentales sur le monde des sons musicaux et de la perception auditive. Les exemplaires de Gérard Grisey portent de nombreuses annotations et parties de texte surlignées. Ils doivent, en ce sens, être inclus dans le dossier génétique car, grâce à eux, le compositeur s'approprie un grand nombre de phénomènes sonores qu'il va explorer à travers sa musique. La recomposition de spectre en est un exemple parmi d'autres.

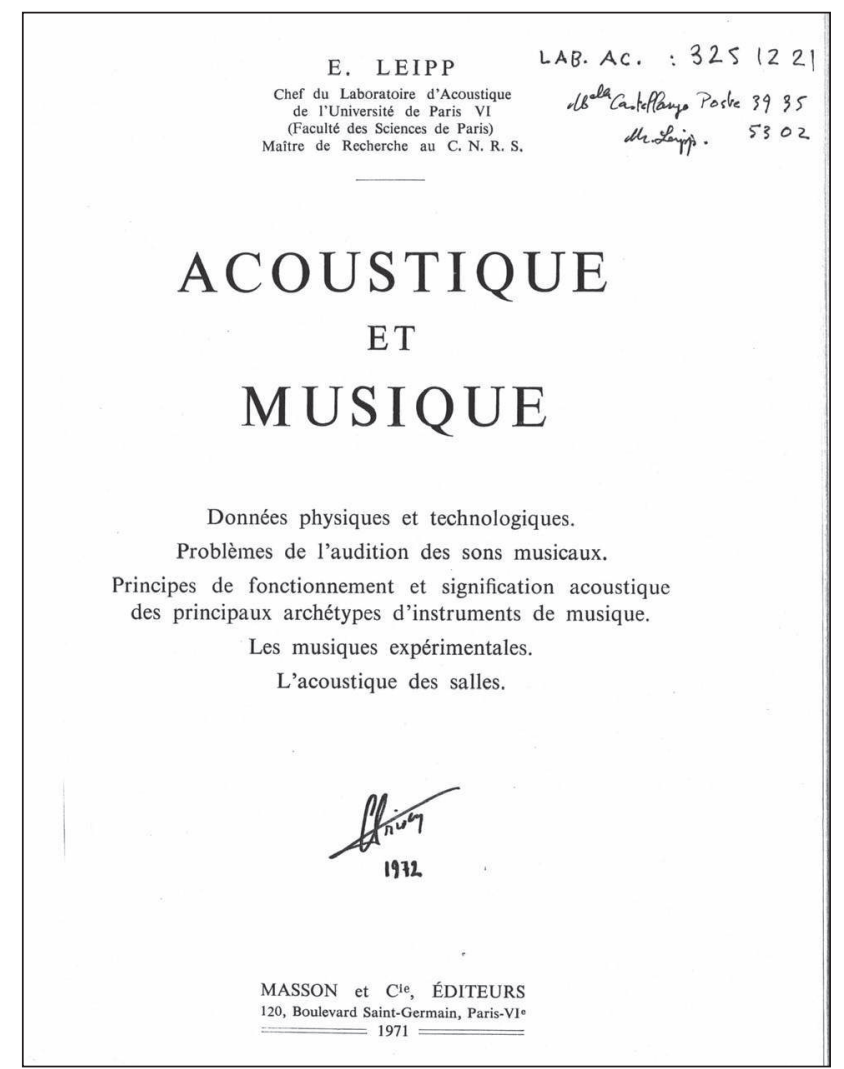

Pour restituer les composantes d'un spectre harmonique, il est nécessaire de faire appel à des hauteurs n'appartenant pas au tempérament égal qui est fondé sur la division de l'octave en douze demi-tons égaux. Les instruments à hauteurs déterminées (tel le piano) ne peuvent restituer qu'un nombre restreint d'harmoniques à moins d'en modifier le système d'accordage. En revanche, tout instrument pouvant produire des micro-intervalles (tel le violon) est susceptible de restituer les hauteurs coïncidant avec les fréquences des composantes d'un

Fig. 2 : Pages de garde (datées et signées par Gérard Grisey)

des ouvrages Acoustique et musique d'Émile Leipp et Vues nouvelles sur le monde des sons de Fritz Winckel.

Au sein du laboratoire d'acoustique (université de Paris VI), Gérard Grisey suit tout d'abord les cours de Leipp (1974-1975) puis collabore étroitement avec Michèle Castellengo pour la réalisation de sonagrammes qu'il utilisera dans Modulations (1976-1977) et Transitoires (1980-1981) (Coll. Gérard Grisey, Fondation Paul Sacher) 
spectre. Mais le son émis n'est pas pur : il est, suivant un mot de Grisey, une «microsynthèse ${ }^{14}$ ». Son spectre peut être qualifié « de microphonique car l'oreille, généralement, ne désigne pas les partiels mais se contente d'une perception globale, ce qu'on appelle le timbre15 ». Par superposition de spectres microphoniques, Grisey façonne un spectre synthétique qu'il qualifie de macrophonique car, cette fois, « les composantes sont bien nommées mais nous tendons à les intégrer dans une perception globale sans parvenir toutefois à la définir ${ }^{16}$ ».

La première ébauche de ce procédé compositionnel est réalisée en 1973 alors que Grisey travaille sur Dérives (1973-1974), mais il n'est cependant pas encore question de micro-intervalles, contrairement à la dernière page de Périodes dans laquelle " l'instrumentation et la distribution des volumes et des intensités suggèrent un spectre synthétique qui n'est autre que la projection dans un espace dilaté et artificiel de la structure naturelle des sons $17 »$. Nous décrirons ce procédé en partant de la première page de la partition de Partiels, qui introduit la première section de l'œuvre. Nous remonterons ensuite dans les documents de genèse qui, en raison de leur teneur scientifique, nécessitent un commentaire détaillé (restituant les implicites des termes et schémas en même temps que leur transcription). Enfin, en retournant à la partition, nous espérons illustrer pleinement le caractère décisif des avant-textes et expliciter la manière dont ils sont transcrits musicalement.

14. Gérard Grisey, «À propos de la synthèse instrumentale » (1979), dans Écrits, op. cit., p. 35 .

15. Gérard Grisey, «La musique : le devenir des sons » (1982), dans Écrits, op. cit., p. 50.

16.Ibid.

17.Ibid., p. 92.

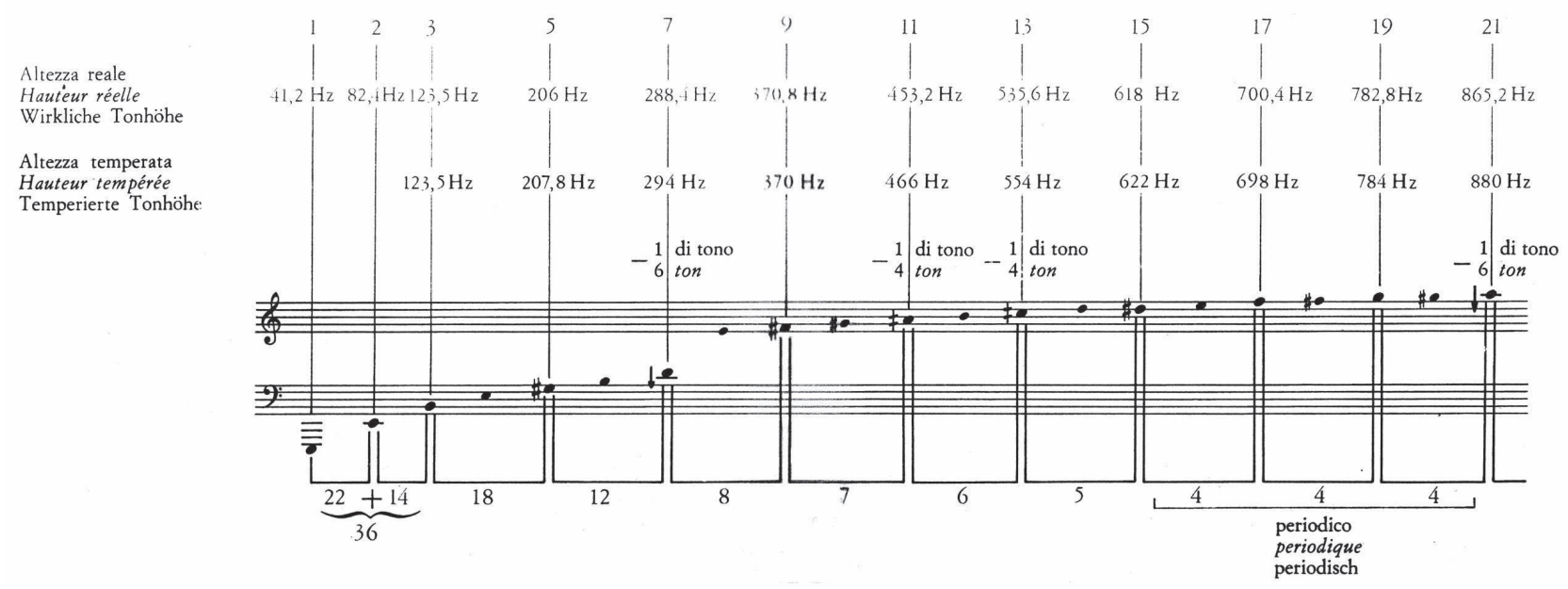

Fig. 3 : Transcription musicale du spectre façonnant l'ensemble des pièces du cycle Les espaces acoustiques. Cette figure apparaît dans la notice de la partition Périodes (Ricordi 132243, 1974). Sur la partie supérieure, le compositeur indique le numéro des harmoniques qu'il a retenus, la fréquence physique correspondante (en Hertz), la fréquence de la hauteur tempérée qui s'en rapproche le plus et enfin, lorsque l'écart est important, le micro-intervalle imputé à la hauteur tempérée pour approcher la fréquence réelle de l'harmonique. Sur la partie inférieure, le compositeur indique la valeur (en quart de tons) de l'intervalle formé par deux notes successives du modèle spectral 
Gérard Grisey

PARTIELS pour 18 musiciens

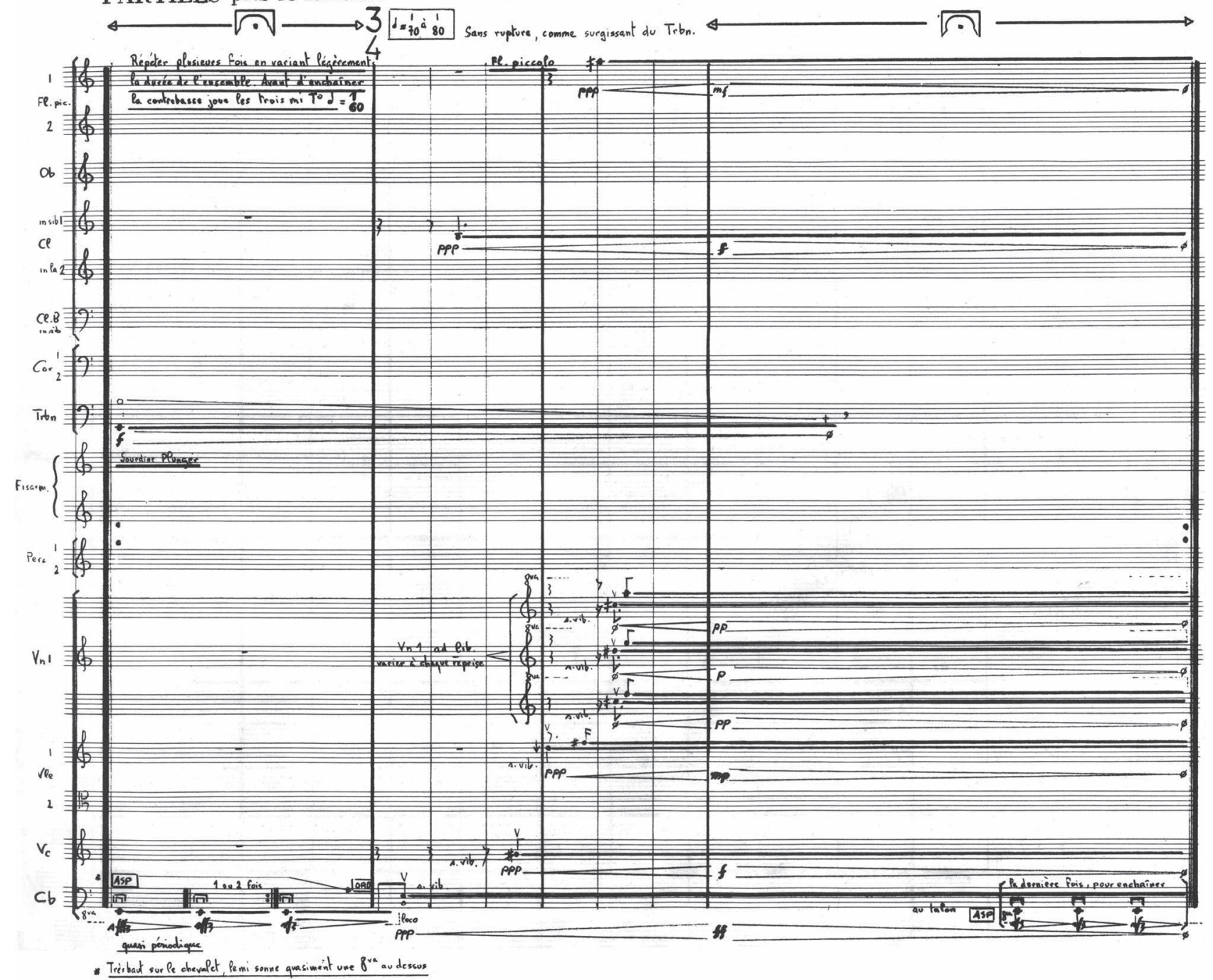

Fig. 4 : Partiels, mesure 1 à 4, première page de la partition (Ricordi 132423, 1976). Alors que la contrebasse joue un motif rythmique « quasi périodique », le trombone reproduit la fondamentale $\left(m i_{1}\right)$ du spectre synthétique en voie de construction (mesure 1 , respectivement première et onzième portées en partant du bas). Les instruments restituent alors à tour de rôle les harmoniques de ce spectre (mesures 2 et 3 ) qui sont maintenus sans modification de leur intensité respective (début mesure 4) avant de disparaître très progressivement (suite mesure 4) 


\section{Le spectre synthétique : trait d'union entre Périodes et Partiels}

Périodes et les autres œuvres du cycle Les espaces acoustiques sont construites autour d'un spectre harmonique que nous qualifions d'originel et dont la fréquence fondamentale est $41,2 \mathrm{~Hz}$, soit $m i_{0}$. Grisey accorde une importance particulière à certaines composantes de ce spectre harmonique : la fondamentale (soit l'harmonique 1), l'harmonique 2 et les harmoniques impairs qui suivent. Dans Périodes, le spectre originel comprend douze composantes que Grisey transcrit approximativement grâce à l'emploi de microintervalles. Il utilise des quarts de dièse $[\ddagger]$ pour restituer les harmoniques 11 et 13 et des inflexions « d'environ $1 / 6$ ton $»[\downarrow]$ pour les harmoniques 7 et 21 (voir fig. 3 ).

La première page de Partiels est identique à la dernière page de Périodes : elle sert de transition lorsque les deux pièces sont interprétées dans la continuité. Cette section commune met en scène la répétition ad libitum d'un spectre synthétique ayant pour fondamentale $m i_{1}$. La construction progressive de ce spectre, composante par composante, vise à retracer la nature temporelle de la plupart des sons musicaux : attaque, maintien et disparition. En acoustique, les termes employés pour décrire ces différents épisodes (visibles sur la figure 1) sont le transitoire d'attaque, phase durant laquelle les différentes composantes spectrales apparaissent suivant d'infimes décalages, le régime stationnaire (ou permanent) généralement stable et enfin, le transitoire d'extinction, phase plus ou moins longue durant laquelle les composantes se dissipent graduellement. En restituant ces trois phases, Grisey établit « une sorte de projection du microphonique sur un écran macrophonique qui suppose un agrandissement non seulement sonore mais temporel18 ».

Deux éléments distincts constituent ce passage : le motif rythmique joué à la contrebasse et l'établissement progressif du spectre synthétique (voir fig. 4). Le trombone, en jouant forte la note $m i_{1}$, pose la fondamentale du spectre synthétique ; en même temps, la contrebasse martèle de façon «quasi périodique » trois ou quatre $m i_{0}$ (l'indication $8 v a$ en dessous de la clé de $f a$ signifie qu'il faut transposer à l'octave inférieure) ${ }^{19}$. Les différents harmoniques apparaissent à tour de rôle par ordre croissant, de manière furtive (nuance $p p p$ ) pour entrer « sans rupture, comme surgissant du trombone ». L'intensité de chaque harmonique ainsi restitué croît progressivement jusqu'à atteindre une valeur maximale (début de la dernière mesure) : cette phase correspond au transitoire d'attaque. L'intensité est alors maintenue quelques secondes : c'est la restitution du régime stationnaire. Enfin, la dissolution de la fondamentale au trombone puis des autres composantes correspond au transitoire d'extinction.

Le choix des hauteurs est conditionné par les numéros des harmoniques du spectre originel (voir fig. 3). Le trombone restitue la fondamentale $m i_{1}$ (mesure 1) ; la contrebasse, la clarinette et le violoncelle, les harmoniques 2,3 et 5 (mesure 2) ; l'alto, les harmoniques 7 et 9 ; la flûte piccolo, l'harmonique 11 ; enfin le violon, les paires d'harmoniques 13-17, 15-19 ou 13-21. La conception spectrale du matériau harmonique, les modes de jeu instrumental (le plus neutre possible, sans vibrato) et les dynamiques employées (fondues partant de ppp pour effacer au maximum les attaques individuelles) concourent à l'annihilation des identités instrumentales au profit d'une sonorité d'ensemble. Le spectre synthétique ainsi créé par Grisey rassemble les timbres individuels en une unité fonctionnelle intégrée dans un phénomène global situé à la frontière entre le timbre et l'harmonie :

Nous venons de créer un être hybride pour notre perception, un son qui sans être encore un timbre, n'est déjà plus tout à fait un accord, sorte de mutant de la musique d'aujourd'hui, issu de croisements opérés entre les techniques instrumentales nouvelles et les synthèses additives réalisées par ordinateur 20 .

18. «Les dérives sonores de Gérard Grisey », entretien avec Guy Lelong (1988), dans Écrits, op. cit., p. 239

19. En précisant qu'il faut jouer très haut sur le chevalet (ASP pour alto sul ponticello), Grisey souhaite que « le $m i$ sonne quasiment une $8^{\text {va }}$ [octave] au dessus », c'est-à-dire à l'unisson du trombone.

20. Gérard Grisey, «La musique : le devenir des sons » (1982), dans Écrits, op. cit., p. 50-51. 


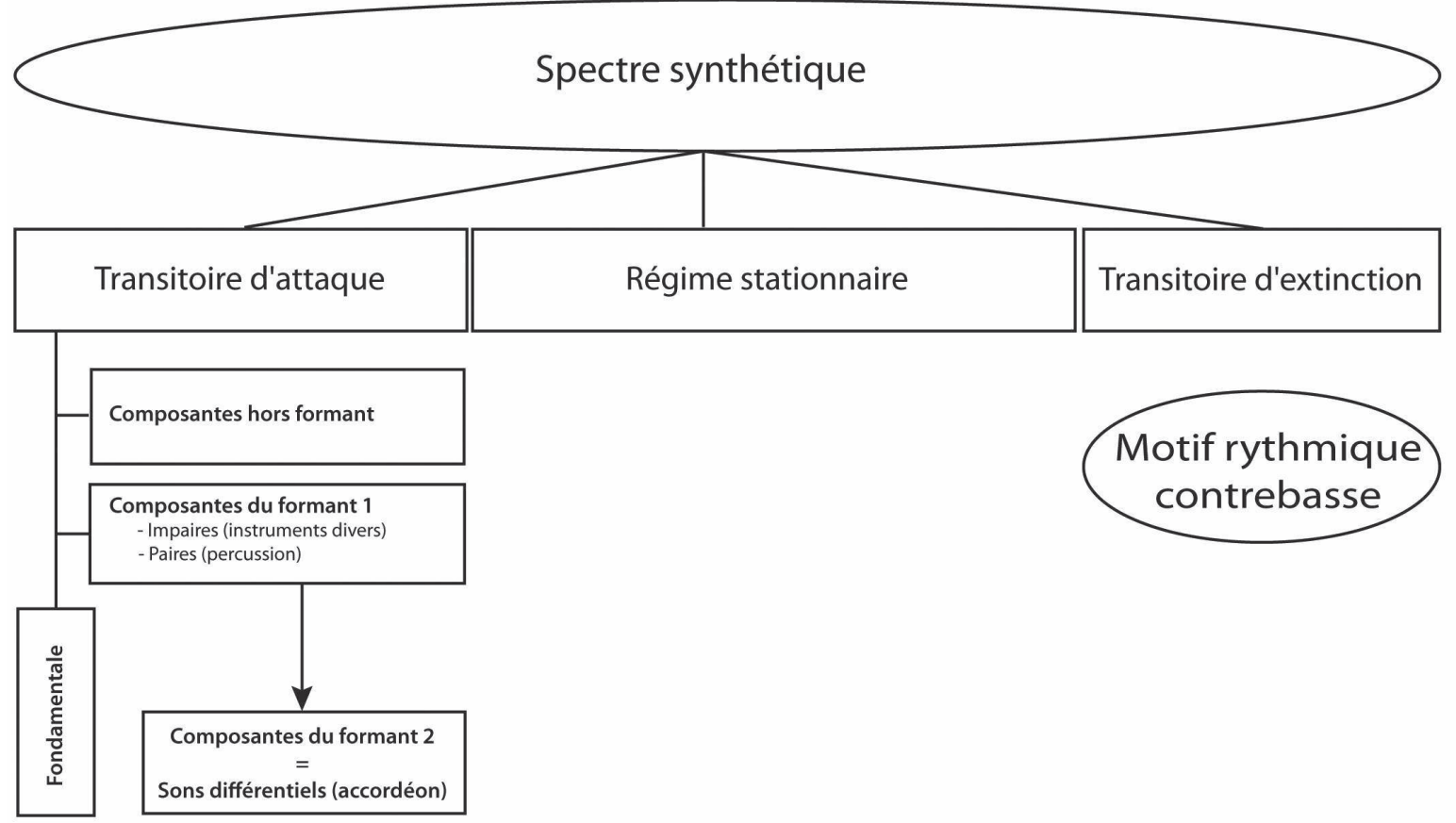

Fig. 5 : Représentation schématique del'entité musicale explorée dans la première section de Partiels. Nous distinguons d'une part le spectre synthétique qui est constitué d'un transitoire d'attaque, d'un régime stationnaire et d'un transitoire d'extinction, d'autre part le motif rythmique confié à la contrebasse durant le transitoire d'extinction. À chaque nouvelle occurrence de cette entité, ces deux éléments subissent un ensemble de modifications parfaitement contrôlées par le compositeur

\section{Les processus de transformation dans la première} section de Partiels

Cette technique de synthèse instrumentale mise en œuvre par Grisey à la fin de Périodes lui «paraissait devoir être développée 21 ». La première section de Partiels, qui débute après la barre de reprise de la première page (voir fig. 4), s'articule autour de la figure musicale que nous venons de décrire et dont la figure 5 donne une représentation schématique détaillée. Cette figure est répétée plusieurs fois mais subit, à chaque nouvelle occurrence, des modifications de plus en plus importantes qui sont gérées par des processus complexes de transformation. 
Un processus, rappelle Philippe Leroux, « représente une façon de tracer des chemins continus d'un événement sonore à un autre 22 ». Il " se présente donc comme une loi ou un système de loi qui régit les flux d'événements sonores, ou la transformation continue d'un objet dans un autre23 ». Dans son analyse de Partiels, Leroux en repère cinq : constitution du spectre suivie de sa détérioration dans le grave, altération de la périodicité du $m i$, rapprochement des transitoires d'attaques, allongements des extinctions, transformations des timbres individuels vers le bruit 24 .

Le spectre synthétique (enrichi du vingtcinquième harmonique) et le motif de contrebasse qui l'annonce sont en effet l'objet de plusieurs processus de transformation guidés par des opérations compositionnelles extrêmement précises : l'objectif du compositeur est de transformer le spectre harmonique et le motif périodique du début en un spectre inharmonique chargé de sonorités bruitistes et associé à un motif rythmique totalement apériodique. Voici comment Grisey résume lui-même ce passage.

Au début de Partiels, le spectre d'harmoniques du mi de la contrebasse et du trombone est actualisé par dix-huit instruments. Ce spectre naturel dérive à chaque répétition vers un spectre de partiels inharmoniques. La zone formantique, progressivement décalée vers le grave, se colore de fréquences de plus en plus inharmoniques. Elle est généralement confiée aux bois sans vibrato et sans diminuendo. Les durées des transitoires d'attaque et d'extinction évoluent elles aussi pour chaque répétition en raison inverse : les transitoires d'attaque croissent, les transitoires d'extinction décroissent. Les durées des zones stables fluctuent autour d'une constante.

Les changements de composantes du spectre, les changements de timbre de chaque partiel et les événements transitoires qui surviennent dans le corps du son sont additionnés. Ils constituent le degré de changement entre un état de l'évolution spectrale et le suivant 25 .
Ces propos peuvent paraître au premier abord relativement abscons mais prennent tout leur sens après une analyse détaillée des avant-textes. Le fonds des manuscrits musicaux de la collection Gérard Grisey (fondation Paul Sacher) consiste en un ensemble de «pochettes » consacrées aux différentes œuvres du compositeur. Le contenu de ces manuscrits s'avère très varié : esquisses musicales, notations textuelles, graphiques, tableaux, schémas... Les deux premiers documents (fig. 6 et 7) que nous allons présenter ont été retrouvés dans la pochette «Dérives» mais se rattachent sans aucune ambiguïté à la première section de Partiels. Les deux autres documents (fig. 8 et 10) sont rangés dans la pochette «Partiels » : s'ils nous en disent peu sur les éventuelles alternatives compositionnelles considérées par le compositeur, ils permettent en revanche d'appréhender les modes opératoires qu'il a mis au point tel un ingénieur, se distinguant résolument du modèle néobeethovénien de la composition comme survenue, variation et développement d'idées mélodiques et harmoniques. Enfin, cet ensemble de documents permet de rendre compte du poids structurel des degrés de changement, des zones formantiques, des sons différentiels..., autant de notions que nous explicitons dans ce qui suit.

21. Note de programme pour Les espaces acoustiques, dans Écrits, op. cit., p. 131.

22. Philippe Leroux, «Intégrer la surprise : les processus dans Partiels de Gérard Grisey ", dans Le Temps de l'écoute : Gérard Grisey ou la beauté des ombres sonores, textes réunis et présentés par Danielle CohenLevinas, Paris, L'Itinéraire/L'Harmattan, 2004, p. 39.

23. Ibid., p. 40.

24. Ibid., p. 41. 25. Gérard Grisey, « Structuration des timbres dans la musique instrumentale» (1991), dans Écrits, op. cit., p. 94.

25. Gérard Grisey, «Structuration des timbres dans la musique instrumentale» (1991), dans Écrits, op. cit., p. 94. 


\section{Fig. 6 - Réflexions sur la transformation du spectre}

Ce feuillet à petits carreaux est scotché à un autre identique (sur sa gauche) sur lequel figure un petit tableau, non rempli (et que nous n'avons pas retenu dans le dossier reproduit ici), comportant comme légendes les lettres « $\mathrm{H} »,\langle\mathrm{~T} »,\langle\mathrm{D} »$ et $\ll \mathrm{I} »$ désignant certainement hauteur, timbre, densité et intensité. Le feuillet présenté ici paraît relever de l'aide-mémoire plutôt que de l'esquisse à proprement parler. Grisey y schématise grossièrement le devenir sonore du début de Partiels et pose par écrit quelques idées pour transformer le spectre initial.

Dans le coin supérieur gauche, il schématise la première section de l'œuvre dans laquelle il souhaite agencer « 13 frappés quasi périodiques », « 11 ou $13 \mathrm{mi}+$ spectres ». Le terme «frappés » anticipe vraisemblablement les notes jouées vigoureusement par la contrebasse dans la partition finale. Par « quasi-périodiques », Grisey se réfère à des événements qui se répètent dans le temps à intervalles qui ne sont pas parfaitement réguliers mais presque : le compositeur poursuit ainsi une idée développée dans Périodes, œuvre dans laquelle des événements périodiques « fluctuent légèrement autour d'une constante, analogue à la périodicité de notre battement de cœur, de notre respiration, de notre marche 26 ».

Le «spectre » synthétique qui est parfaitement harmonique au départ doit glisser vers le «bruit» par le biais non pas d'un «bruit variable » mais d'un « formant variable » qui va se décaler de l'« aigu » vers le « grave ». Un formant (ou zone formantique) désigne une plage fréquentielle à l'intérieur d'un son où les composantes spectrales sont particulièrement intenses 27 . La partie droite de ce premier schéma décrit un retour progressif vers le « spectre » avec des notes plutôt « tenue[s] » et une montée progressive de la texture sonore dans les aigus : elle concerne la seconde section de Partiels. Dans son ensemble, l'œuvre joue sur un balancement permanent entre ces deux pôles fort contrastés que représentent le spectre harmonique d'une part et le bruit d'autre part. Quant aux calculs dans le coin supérieur droit de la page, ils se rapportent principalement à des durées envisageables de sections selon le mode opératoire utilisé par Grisey dans Périodes : multiplication par « 8 » des intervalles formés par deux harmoniques conjoints dans le spectre originel (voir fig. 3).
Au fil des onze occurrences du spectre synthétique, le compositeur projette de rendre de plus en plus présent (« -- - + ++ +++ ») les « A) battement [et] son différentiel ». Ces deux phénomènes acoustiques se manifestent lors de l'émission simultanée de plusieurs sources sonores. Si par exemple deux sons purs de fréquence voisine (différence de quelques Hertz) sont émis simultanément, nous n'entendons qu'une seule et unique hauteur agrémentée d'une modulation périodique de l'amplitude ressentie comme des battements : le nombre de battements par seconde correspond à la différence fréquentielle 28 . Lorsqu'il dépasse la trentaine, " on commence à entendre un véritable son supplémentaire $29 »$ correspondant au premier différentiel : la hauteur de ce son évanescent correspond à la différence fréquentielle entre les deux signaux émis. Bien que la perception de ces phénomènes soit considérablement amoindrie lorsqu'ils sont issus de sons complexes ( $v s$ de sons purs), ils demeurent présents. Les ouvrages d'acoustique musicale ne manquent pas de développer ces deux aspects. Grisey envisage aussi de rendre les «B) Transitoires d'attaque » de plus en plus audibles au fil du temps («0 - + ++ seul[ement] transi[toire] »). Quant au « Formant », il précise qu'il y en a « 2 distant/aigu/suraigu » au départ mais qu'à l'arrivée, il n'y en a plus qu' « 1 seul grave».

Le croquis situé au milieu de la page décrit le principal objet compositionnel de toute cette section, i.e. le spectre synthétique, selon une représentation de type sonagraphique (voir Fig. 1). Grisey précise les différentes phases : «(1) attaque », soit la fondamentale du spectre, « (2) établissement du spectre et de son formant », soit les autres composantes spectrales, «(3) 》 le régime stationnaire, et enfin «(4) transitoire d'extinction ». L'attaque et l'établissement du spectre constituent le « $\underline{\text { transitoire }}$ »'attaque.

26. Gérard Grisey, « Tempus ex Machina » (1982-1987), dans Écrits, op. cit., p. 64.

27. La notion de formant est largement développée dans l'ouvrage de Fritz Winckel, Vues nouvelles sur le monde des sons, op. cit. Grisey a d'ailleurs inscrit « $\underline{\text { important } ~}$ à la page 13 de son exemplaire, juste à côté du paragraphe intitulé « La théorie formantique du timbre ».

28. Pour s'accorder entre eux, les musiciens jouent à l'unisson et minimisent justement ces battements pour être exactement sur la même fréquence fondamentale.

29. Émile Leipp, Acoustique et musique, op. cit., p. 28. 


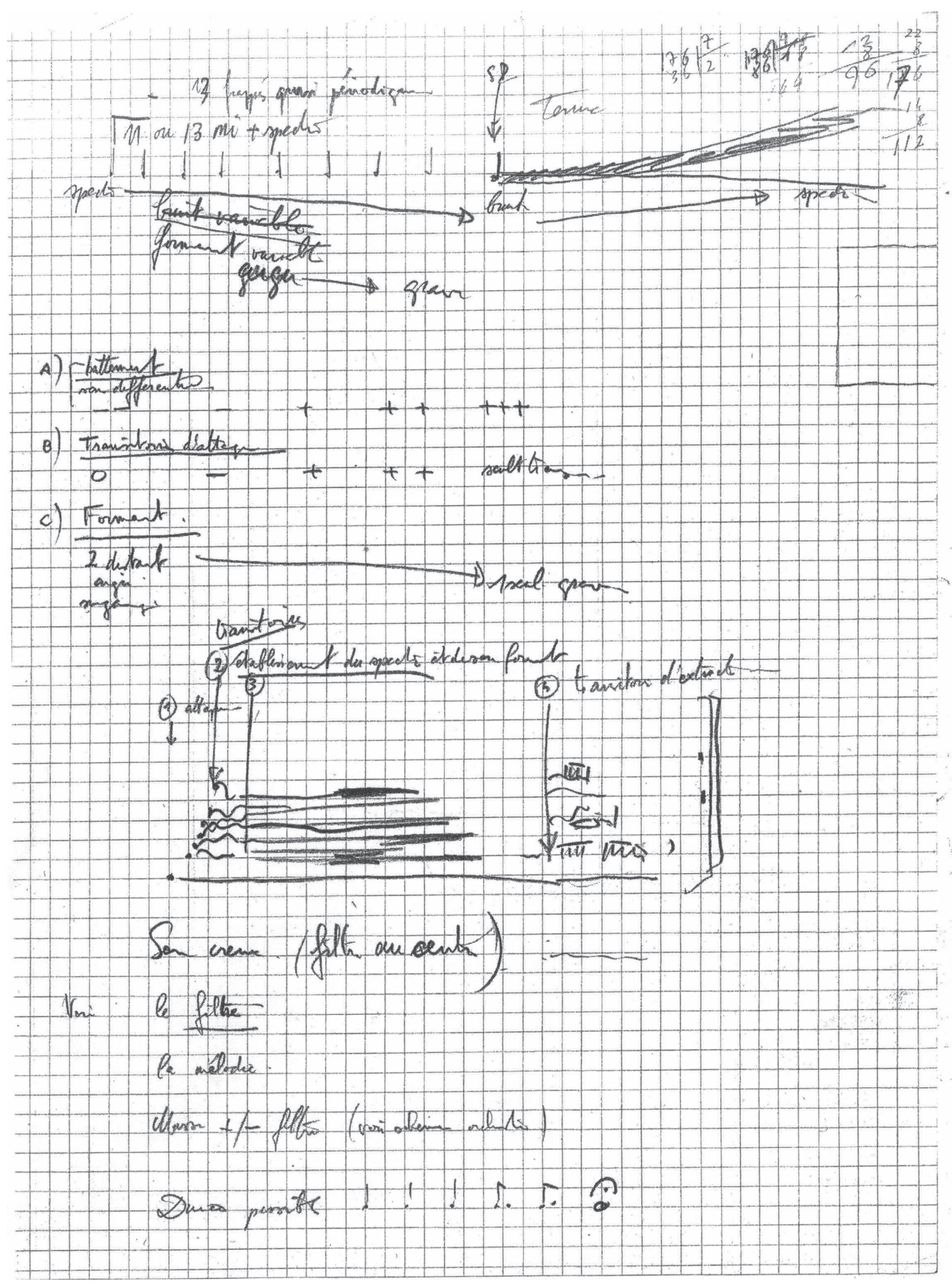

Fig. 6 : Esquisse réalisée au crayon sur une feuille petits carreaux (format $29 \times 20 \mathrm{~cm}$ ). Grisey note des idées concernant la transformation du spectre synthétique (Coll. Gérard Grisey, pochette « Dérives », Fondation Paul Sacher) 
L'indication « Son creux (filtré au centre) » située juste en dessous du croquis renforce l'idée de deux formants distants. Leipp emploie lui-même l'adjectif « creux » dans Acoustique et musique 30 pour décrire certains sonagrammes, ce qui nous conforte dans l'idée que ce livre a exercé une influence notable sur la genèse de cette technique compositionnelle. Les autres notations en bas de cette esquisse, « voir le filtre/la mélodie/Masse +/- filtré (voir schémas orchestres)/Durées possibles », se présentent comme une liste d'idées sur lesquelles réfléchir.

En somme, ce document détermine les traits de l'idée principale qui va guider la totalité de la première section. Un spectre synthétique, de fondamentale mi, sera à chaque nouvelle occurrence modifié (en altérant les formants, les transitoires d'attaque et les sons différentiels qui le définissent) pour obtenir quelque chose de bruitiste au bout de onze ou treize itérations. Une première tentative de mise en place des opérations compositionnelles figure au dos ce document.

\section{Fig. 7 - Mise en place des modes opératoires}

Les notations et calculs figurant sur cette esquisse se rapportent aux sons différentiels et aux densités de changement. Sur la partie supérieure du document, Grisey note quelques-unes de ses découvertes acoustiques :

(Les harm.[oniques] paires peuvent apparaître comme différentiels de 2 harm.[oniques] impairs ff [nuance fortissimo] (formant)

(Les partiels non harm[oniques] ne peuvent pas être engendrés par le spectre.

Il faut un ajout volontaire

transitoire non stabilisé par exemple d'attaque

(Voir spectre à partiels en dim.[inuendo] cf piano)

Grisey schématise alors l'enveloppe temporelle d'un spectre synthétique (la fréquence est représentée verticalement et le temps horizontalement) : le transitoire d'attaque est plutôt court et abrupt, le régime stationnaire parfaitement stable et le transitoire d'extinction long et sinueux. Les calculs figurant sur la partie droite du document sont des manipulations sur les fréquences et numéros d'harmoniques. Les calculs barrés établissent la fréquence du premier son différentiel produit par chacun des onze intervalles formés par les harmoniques conjoints du spectre originel de Périodes (voir fig. 3). Les autres calculs, situés dans le coin droit, ont sûrement été rajoutés ultérieurement car ils se rapportent aux premiers sons différentiels (produits par les notes du formant principal) que l'accordéon sera amené à restituer.

Sur la partie inférieure du document, Grisey établit un tableau (qu'il ne remplit que partiellement) avec en ordonnée les différents paramètres sur lesquels peuvent agir les «Densités de Chgts [changements] » qui, globalement, sont croissantes. Cette notion de degré ou densité de changement est apparue essentielle pour Grisey, alors qu'il était encore étudiant au conservatoire de Paris.

Qu'est-ce qui se passe lorsque je couche sur le papier un timbre, un accord? Il me fallait repartir à zéro, faire une sorte de tabula rasa de tout mon savoir. Quand on s'interrogeait sur les fondements de la perception, on arrivait très vite au phénomène que Stockhausen appelait degré de changement, qui provient de la théorie de l'information, Stockhausen en avait longuement parlé lorsqu'il était venu dans la classe de Messiaen pour analyser Carré [œuvre pour quatre orchestres et quatre chœurs composée en 1959-1960]. Il disait : « quand j'écris un accord, puis un autre, je me demande ce qui a changé entre les deux ». Autrement dit, l'important n'est pas que l'accord soit constitué de tel ou tel intervalle, mais qu'il engendre le degré de changement ${ }^{31}$.

Dans la notice de la partition de Périodes, Grisey revendique clairement cet aspect de son artisanat compositionnel. « Tenir compte de la relativité de la perception : si la musique est le devenir du son, plus que l'objet sonore lui-même, il faudra contrôler sa métabole, ce que je nomme son "degré de changement" autrement dit son voyage dans le temps, son aventure 32 . » Pour la première section de Partiels, ces degrés sont définis par un nombre se rapportant aux changements à opérer entre une occurrence donnée d'un spectre synthétique et celle qui la précède. Le «Spectre imp[air] » est « Fixe » au cours des premières occurrences; rien n'est précisé

\section{Ibid., p. 151 .}

31. Gérard Grisey, entretien avec Danielle Cohen-Levinas (12 décembre 1989), cité par D. Cohen-Levinas, « Gérard Grisey : du spectralisme formalisé au spectralisme historisé », dans Vingt-Cinq Ans de création musicale contemporaine : l'Itinéraire en temps réel, textes réunis et présentés par D. Cohen-Levinas, Paris, L'Itinéraire/L'Harmattan, 1998, p. 53. 32. Notice de la partition Périodes (Ricordi 132243, 1974). 


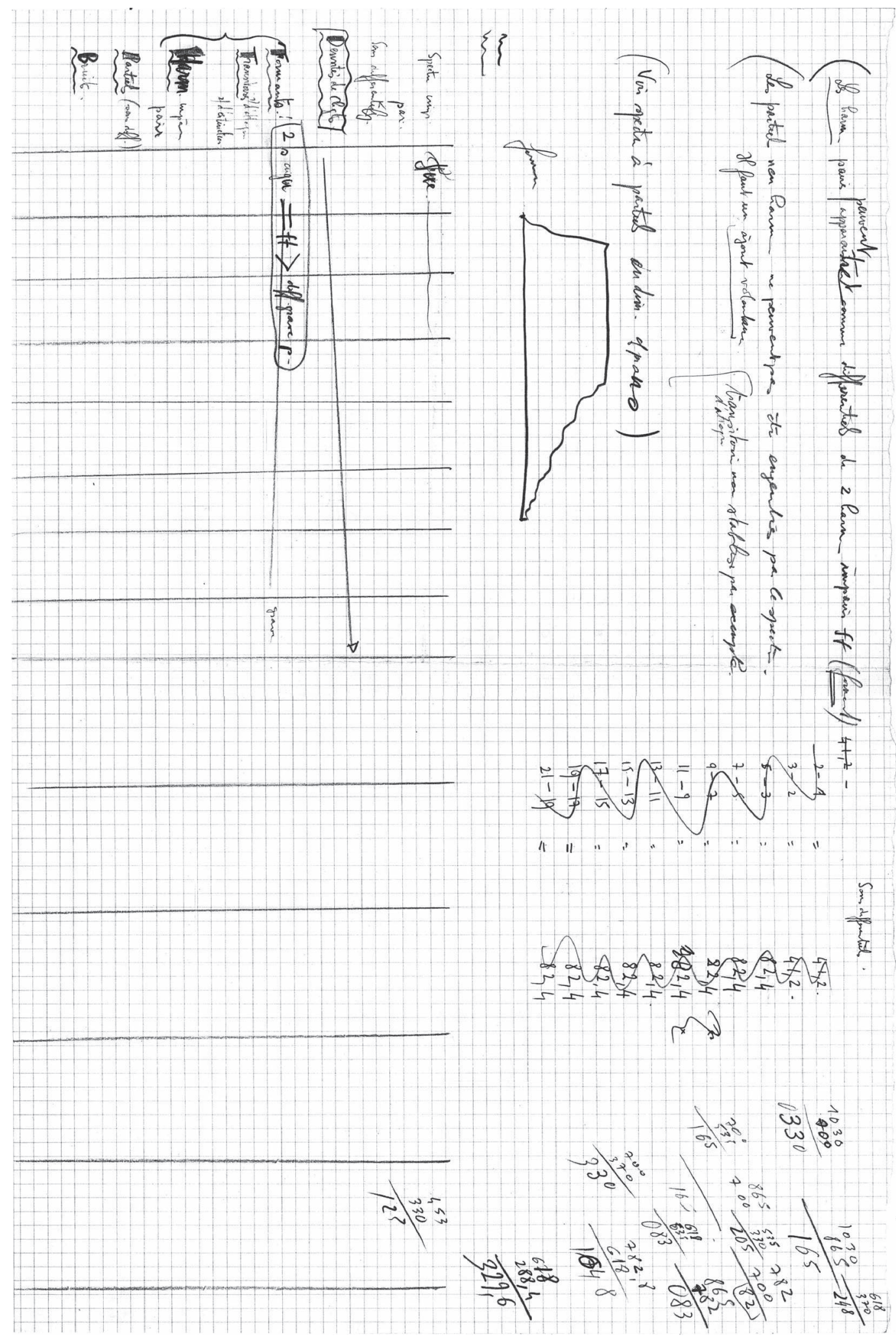

Fig. 7 : Esquisse au crayon et stylo noir sur deux feuilles petits carreaux scotchées entre elles (format $58 \times 20 \mathrm{~cm}$ ). Grisey effectue des calculs de sons différentiels et indique des paramètres sur lesquels pourraient s'exercer les densités de changement.

Le verso de la partie droite correspond à l'esquisse précédente (Coll. Gérard Grisey, pochette « Dérives », Fondation Paul Sacher) 
pour le spectre « pair» ni pour les « Sons différentiels ». Les « « 2 s aigu=ff $>$ diff grave p. », Grisey signale que deux notes aiguës peuvent engendrer, lorsqu'elles sont jouées très fort (nuance fortissimo) un son différentiel grave faible (nuance piano). Aucune évolution n'est mentionnée pour le reste des paramètres : « Transitoires 1) d'attaque/ 2) d'extinction », « $\underline{\text { Harm }}[$ onique] impair/pair », « $\underline{\text { Partiels }}$ (son diff[érentiel]) », « Bruit ».

Nous pouvons supposer que le compositeur avait envisagé de noter l'évolution des changements à opérer sur ces différents paramètres, mais qu'il préféra établir une esquisse davantage structurée afin de contrôler minutieusement la structure de chaque spectre synthétique. Le document suivant (voir fig. 8) est en ce sens remarquable car, tel un plan architectural étalé sur la table de travail, il permet au compositeur de bâtir précisément chacune des occurrences du spectre synthétique tout en gardant une vue d'ensemble sur le devenir sonore.

\section{Fig. 8 - Métamorphose du spectre synthétique}

Depuis Dérives, Grisey conçoit un grand nombre de ses esquisses en assemblant du papier petits carreaux (partie supérieure) avec du papier musique (partie inférieure), obtenant ainsi d'immenses plans de travail qu'il est possible de mettre bout à bout pour revenir sur le déroulement musical en train de se construire. Telle une partition graphique, la section supérieure, authentique lieu de détermination des principaux aspects de la composition, lui permet de concevoir aisément la structure temporelle des différentes sous-sections, les opérations effectuées sur les différents paramètres sonores (densité de changement) ainsi que l'évolution des tessitures extrêmes (l'enveloppe spectrale de sa musique en quelque sorte). La section inférieure de l'esquisse, ultime étape avant la réalisation du brouillon, est en quelque sorte une mise en musique de la partie supérieure.

La partie gauche de la figure 8 représente une de ces esquisses dans sa totalité (format $64 \times 49 \mathrm{~cm}$ ) alors que la partie droite qui en est la suite fait partie d'une autre esquisse (la zone manquante a été volontairement coupée car elle concerne le début de la seconde section de Partiels). Nous nous limiterons à une présentation des quatre légendes de la partie supérieure du document (voir fig. 9) qui se rapportent respectivement aux «Durées », à la « DENSITÉ de Chgt. [changement] TOTALE », au « REGISTRE - COURBE GÉNÉRALE » et enfin, au détail des «DENSITÉS de CHGTS [changements] ».

L'indication dans le coin supérieur gauche «180" » correspond à la durée totale (exprimée en secondes) de la première section de Partiels (soit environ un septième de l'œuvre). L'indication « $|-|=1$ " » nous renseigne sur l'échelle choisie par le compositeur : chaque carreau est équivalent à une seconde de musique. Dans la ligne portant la légende « Durées », Grisey indique, pour chaque occurrence, les durées des trois phases du spectre ainsi que sa durée totale (juste au-dessus de la ligne). L'unité de référence n'est plus la seconde mais la double croche. Étant donné que le tempo est fixé à la noire $=60$, une double croche équivaut à un quart de seconde si bien que chaque carreau équivaut à quatre double croches. La durée des spectres oscille entre soixante et une et soixante-dix double croches, ce qui illustre parfaitement l'idée de pseudopériodicité (ou périodicité floue) chère au compositeur. Les durées à l'intérieur de chaque spectre sont aussi soumises à des variations. Les durées du régime stationnaire (trente-six, quarante ou quarante-quatre double croches) fluctuent bien autour d'une constante ; la durée du transitoire d'attaque tend à devenir de plus en plus courte (de seize double croches dans la première occurrence à quatre dans la dernière), alors que celle du transitoire d'extinction est allongée (de onze à dix-huit double croches). Les durées totales des phases transitoires d'une part, et des phases stationnaires d'autre part, sont rapportées par le compositeur dans le coin gauche ( total Ta et Te $=69$ " $/$ Milieu $=$ 111 ”»), « Ta » et « Te » se référant respectivement aux transitoires d'attaque et d'extinction et « milieu » à la phase stationnaire. Quant à «> SD », abréviation présente aussi sur le schéma en haut de la figure 6, à la jonction entre la première et la seconde section de Partiels, nous pensons sans certitude qu'elle pourrait signifier spectre diffracté.

La ligne suivante, portant l'indication « DENSITÉ TOTALE de Chgt. » sert à inscrire les densités de changement opérées entre chaque occurrence successive. Le premier spectre étant le point de départ de cette longue mutation, aucun changement ne lui est associé. 


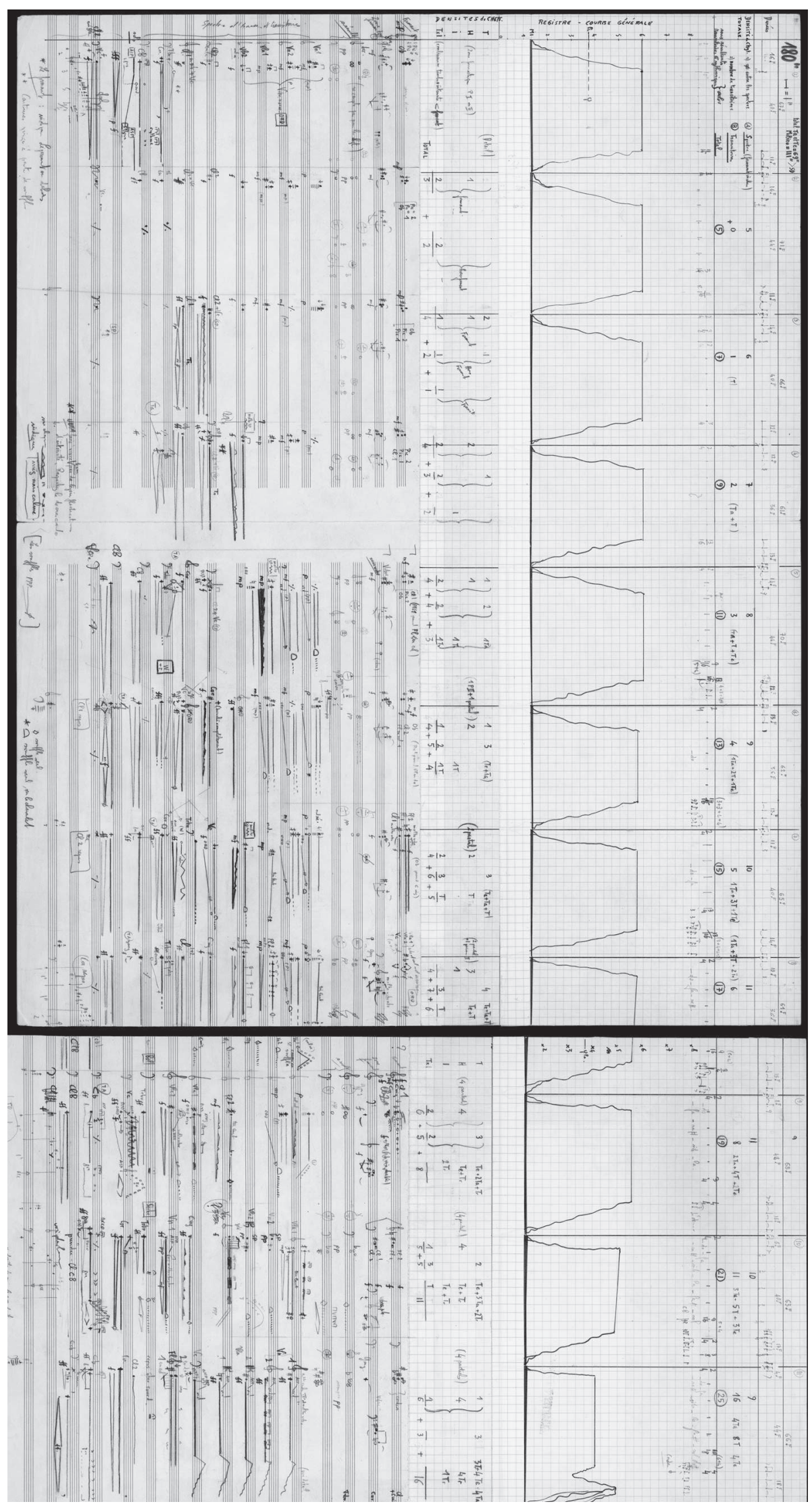

Fig. 8 : Esquisses au crayon, stylo et feutres de couleur sur papier petits carreaux et papier musique. La partie gauche du document correspond à une esquisse intégrale (format $64 \times 49 \mathrm{~cm}$ ) alors que la partie droite correspond à un document similaire mais tronqué (format $29 \times 49 \mathrm{~cm}$ ) afin de ne garder que ce qui se rapporte à la première section de Partiels. Cette figure retrace l'ensemble des modifications apportées au spectre synthétique (Coll. Gérard Grisey,

pochette « Partiels",

Fondation Paul Sacher) 


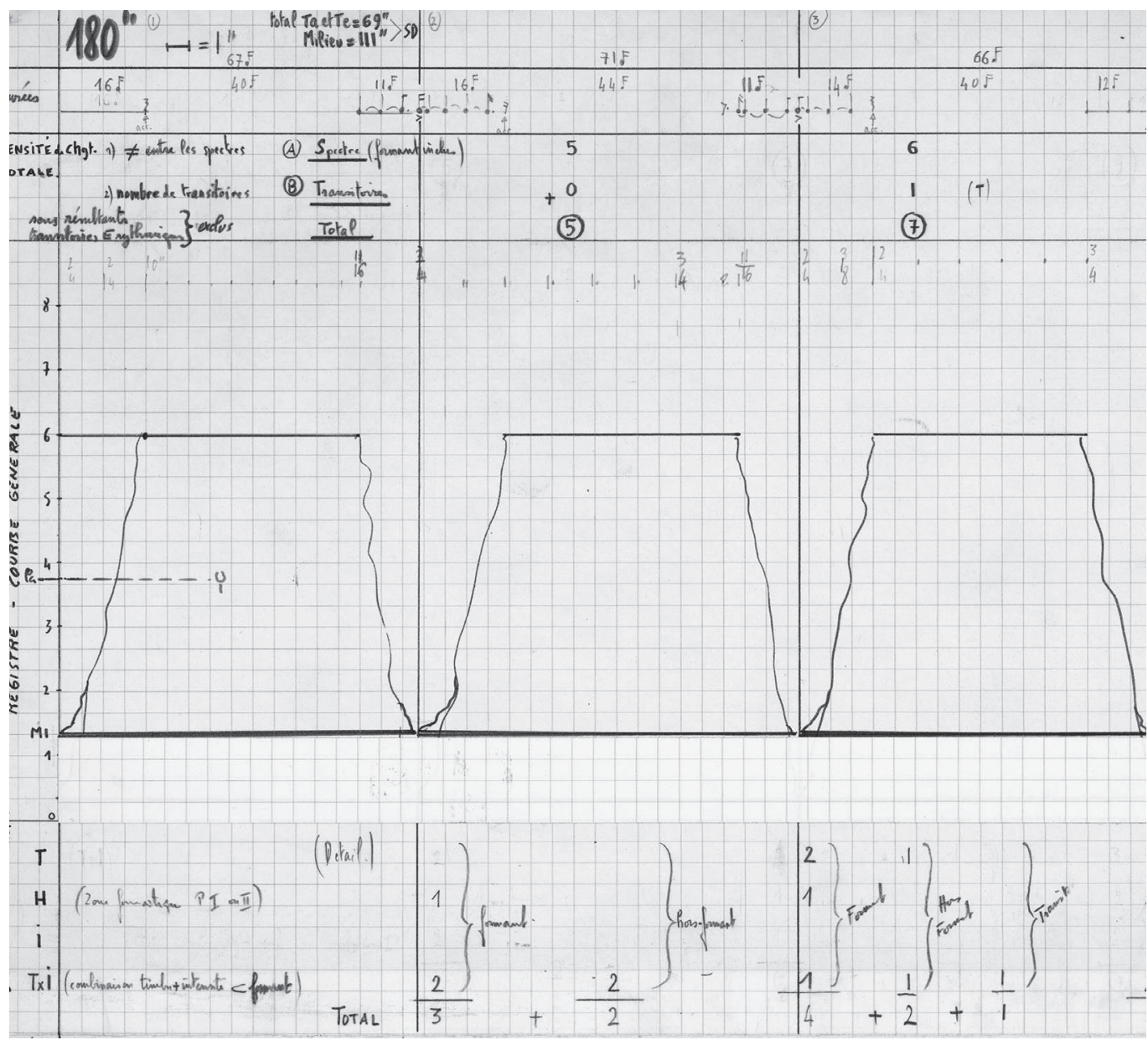

Fig. 9 : Détail (coin supérieur gauche) de la figure 8 (Coll. Gérard Grisey, pochette « Partiels », Fondation Paul Sacher)

Grisey profite de cet espace libre pour apporter quelques précisions. Il distingue sans ambiguïté deux éléments :

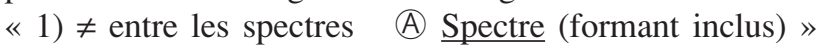
et «2) nombre de transitoires (B) = Transitoires ». Dans la première catégorie, sont comptabilisées les différences portant sur les composantes du spectre, y compris celles du formant. Dans la seconde catégorie, sont comptabilisés, non pas des différences, mais des éléments transitoires à l'intérieur de l'occurrence, i.e. des fluctuations temporelles associées aux différentes composantes du spectre (voir fig. 11 pour la réalisation musicale). Le terme « transitoire » utilisé par Grisey prête à confusion car il se rapporte aussi aux phases d'attaque et d'extinction du son. L'inscription « sons résultants/transitoires E rythmiques » « $\}$ exclus » implique que ces degrés de changement ne portent a priori ni sur la partie d'accordéon qui restitue des sons différentiels issus du formant principal, ni sur le motif rythmique de la contrebasse jouant la note $m i_{0}$ (la lettre « $\mathrm{E} »$ désignant la note $m i$ dans le système allemand $\left.{ }^{33}\right)$. La

33. Grisey a fait une partie de ses études musicales en Allemagne avant d'intégrer le conservatoire de Paris ; aussi utilise-t-il parfois la convention solfégique allemande/anglo-saxonne dans laquelle les notes sont désignées par des lettres $(A, B, C \ldots)$. et non des syllabes $(l a, s i, d o \ldots)$. 
somme « $\underline{\text { Total } » ~ d e s ~ d e u x ~ c a t e ́ g o r i e s ~ d e ~ c h a n g e m e n t ~ e s t ~}$ systématiquement reportée par des nombres encerclés. Par exemple, le second spectre présente cinq différences par rapport au premier mais n'est agrémenté d'aucun élément transitoire; le troisième spectre présente six différences par rapport au second, auxquelles s'ajoute un élément transitoire, ce qui amène la densité totale de changement à $7 ; \ldots$ et ainsi de suite jusqu'au onzième spectre qui présente neuf différences par rapport au dixième et contient seize éléments transitoires, ce qui amène la densité totale de changement à 25 . Le devenir sonore du spectre synthétique, dans ses énonciations successives, est entièrement guidé par cette densité de changement croissante $(5,7,9,11$, $13,15,17,19,21,25)$ dont les nombres sont tirés de la série des harmoniques restitués lors de la première occurrence du spectre. Toute la première section de Partiels est donc construite autour de ce fil d'Ariane qui resterait insoupçonnable sans se prêter à une analyse des esquisses.

La ligne portant l'indication « REGISTRE - COURBE GÉNÉRALE » est la plus spacieuse car elle sert à représenter l'enveloppe temporelle de chaque spectre, forme que le compositeur avait préalablement projeté d'utiliser (voir fig. 7). Grisey ne cherche pas à représenter rigoureusement les durées respectives des différentes phases temporelles : il ne fait que les schématiser. En revanche, les hauteurs inscrites verticalement sont prises en considération : trois carreaux correspondent exactement à une octave. Dans la première occurrence, « la - - - $-\Psi$ - $\Psi$ indique le $l a_{3}$, note de référence que donne le diapason dont les deux branches sont schématisées par le symbole « $\Psi$ ». Ces enveloppes temporelles retracent clairement la succession des onze spectres synthétiques dont la note la plus grave est constamment $m i_{1}$ (fondamentale signalée par un trait noir). À l'autre extrême, la note la plus aiguë du spectre reste au cours des cinq premières occurrences $d o_{6}$ (approximativement l'harmonique 25) ; elle est ensuite systématiquement décalée vers le grave ce qui a pour effet de compresser le spectre. Les numéros plus effacés apparaissant au-dessus de ces formes (juste en dessous de la ligne rapportant les densités totales de changement) concernent la métrique, c'est-à-dire le type et la durée des temps dans les mesures.

La dernière ligne, dont la légende est «DENSITÉ de CHGTS », rapporte le détail de ces changements qui, selon les légendes, s'opèrent sur « $\mathrm{T} », \ll \mathrm{H} »$, et «I », abréviations désignant respectivement le timbre, la hauteur et l'intensité d'un son ; «TxI (combinaison timbre + intensité $\subset$ formant) » se rapporte logiquement à des changements de timbre et/ou d'intensité à l'intérieur du formant principal (le symbole mathématique $\subset$ signifiant «inclus »). À côté de « $\mathrm{H} »$ apparaît « (zone formantique $\mathrm{P}$ I ou II) » : la lettre « $\mathrm{P}$ » se réfère au prisme, terme que Grisey emploie dans les manuscrits de cette époque pour désigner une partie d'un spectre (dans le cas présent, P I et P II se réfèrent respectivement aux spectres d'harmoniques pairs et impairs construits à partir de la fondamentale $m i_{1}$ ). Grisey indique alors pour chaque occurrence le nombre de différences à l'intérieur du « formant » (première accolade) et à l'extérieur de celuici (mention « hors formant» sur la seconde accolade) : la somme totale de ces différences correspond bien à celle reportée dans la partie supérieure de l'esquisse au niveau de «(A) $\underline{\text { Spectre }}$ (formant inclus) ». La troisième accolade, «Transit[oires] », qui n'apparaît qu'à partir de la troisième occurrence, se rapporte au nombre d'éléments transitoires. À partir de la sixième occurrence, Grisey introduit systématiquement des notes étrangères à l'intérieur du spectre synthétique, l'objectif étant de le rendre excessivement inharmonique à la fin de la section : la mention « 1 p. II +1 partiel » pour l'occurrence 6 signifie qu'il faut introduire un partiel et une composante paire dans le spectre synthétique (voir fig. 11).

Le caractère scientifique et synoptique de ces documents illustre une facette importante de l'artisanat du compositeur. Tel un ingénieur ou un architecte, Grisey planifie, avec une extrême rigueur, la structure de chaque spectre synthétique avant de passer à la réalisation musicale. Muni de tels documents, le compositeur est en mesure de rédiger la partition jusque dans la plupart de ses détails. «Quant aux rythmes de la contrebasse, ils proviennent d'un monnayage progressif de la périodicité initiale », indique Grisey dans un de ses écrits théoriques ${ }^{34}$. L'esquisse suivante permet de mieux comprendre la teneur de ces propos.

34. Gérard Grisey, « La musique : le devenir des sons » (1982), dans Écrits, op. cit., p. 51. 


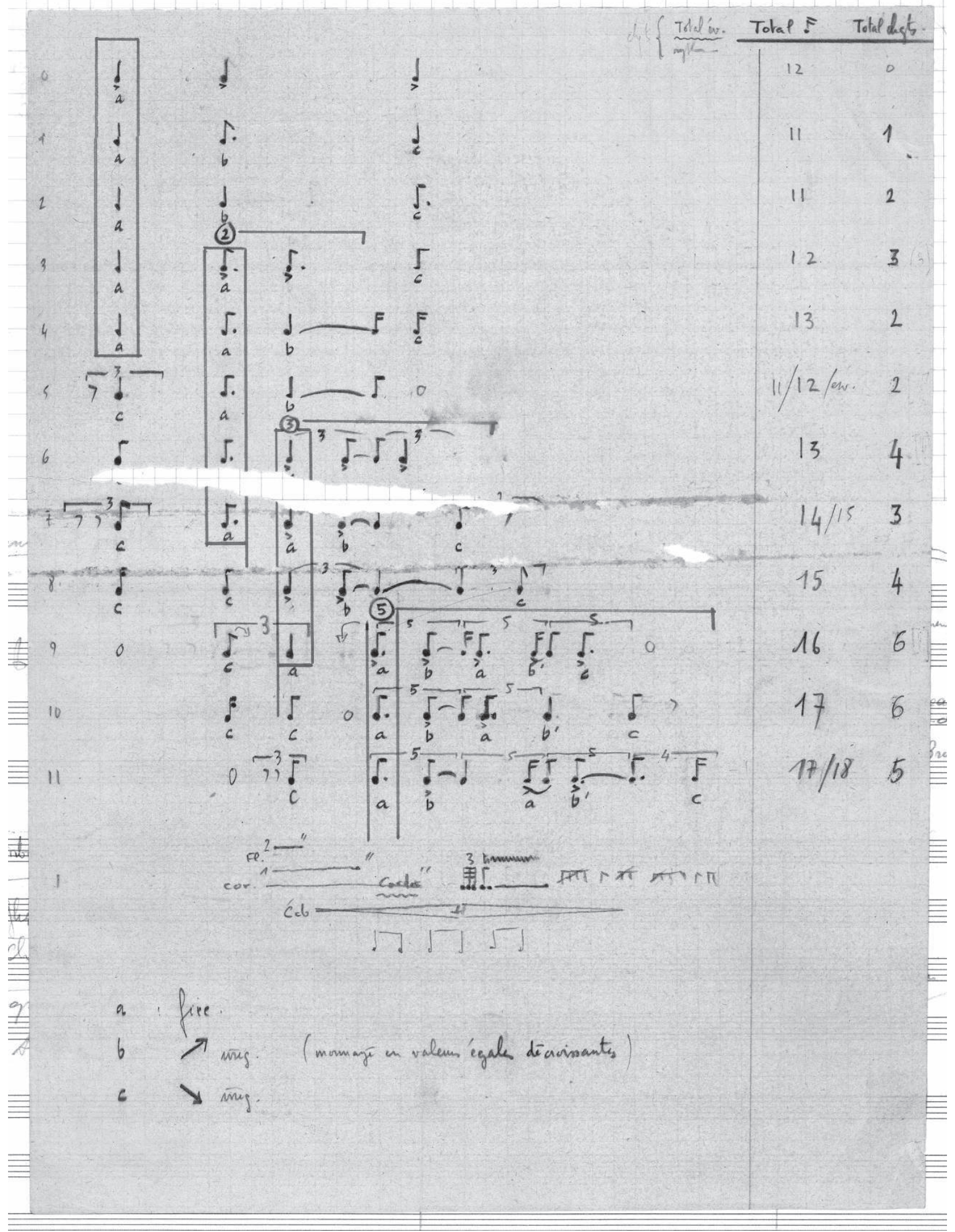

Fig. 10 : Esquisse collée au dos de la seconde partie de la figure 8 et annotée au crayon, stylo et feutre rouge (format $28,2 \times 21,2 \mathrm{~cm}$ ). Ce document relate le processus de transformation opéré sur le motif rythmique de la contrebasse (Coll. Gérard Grisey, pochette « Partiels », Fondation Paul Sacher) 


\section{Fig. 10 - Métamorphose rythmique du motif de la contrebasse}

Le motif rythmique joué à la contrebasse subit un autre processus de transformation. Cet aspect de la conception est traité à part sur un document exclusivement consacré aux « frappés quasi-périodiques » précédemment notés par le compositeur (voir fig. 6). La feuille représentée sur la figure 10 est collée au dos de l'esquisse rapportant la fin de la première section de Partiels (seconde partie de la figure 8).

Grisey précise verticalement sur le bord gauche les numéros des occurrences. Le point de départ (occurrence 0) est la répétition des trois noires avant le début de la première section (voir Fig. 4 le dernier groupe de trois notes à la contrebasse avec l'indication « la dernière fois pour enchaîner »). Le motif de la contrebasse est systématiquement inscrit à l'intérieur du transitoire d'extinction du spectre synthétique, mais il annonce clairement l'arrivée d'une nouvelle occurrence de ce spectre. Le compositeur emploie ce que nous appelons trois fonctions duratives $(\mathrm{a}, \mathrm{b}, \mathrm{c})$, qui agissent sur la durée individuelle des notes du motif et, par voie de conséquence, sur la structure rythmique de l'ensemble. En bas de l'esquisse, Grisey précise « a : fixe », «b $\nearrow$ irrég. » (croît irrégulièrement) et « $\mathrm{c} \searrow$ irré. » (décroît irrégulièrement) ; l'ensemble est « (monnayé en valeurs égales décroissantes) ». Ces fonctions agissent verticalement (Grisey a encadré les séries de valeur constante induites par la fonction a). Toutes les trois occurrences, il rajoute une ou plusieurs notes de sorte à réitérer le processus de transformation qu'il a opéré durant les occurrences [0-1-2]. Mais le processus débute alors sur des valeurs de notes sans cesse plus courtes (noire à l'occurrence $0,3 / 4$ de noire à l'occurrence $3,2 / 3$ de noire à l'occurrence $6,3 / 5$ de noire à l'occurrence 9). Cette opération, qu'il décrit comme un monnayage par valeur décroissante, confère un aspect fractal à la logique compositionnelle.

Sur la partie droite du document figurent la durée totale du motif (exprimée une fois de plus en double croches) et le nombre de différences « Total chgts [changements] » entre une occurrence et sa précédente. Ce nombre se rapporte exclusivement à un changement de valeur rythmique ou à l'intrusion d'une nouvelle note entre deux occurrences successives (la disparition d'une note n'est pas prise en compte en revanche).

\section{Retour à la version publiée}

Pour conclure cette lecture génétique de la première section de Partiels, nous allons décrire, en nous basant sur la partition, la sixième occurrence du spectre synthétique et du motif de la contrebasse qui l'annonce (voir fig. 11). Nous espérons ainsi mettre en lumière, et cela de manière concrète, les correspondances entre les esquisses et la partition finalisée. Nous avons sélectionné cette occurrence car elle correspond au milieu de cette première section et représente un point névralgique dans les processus de métamorphose : pour la première fois, Grisey introduit un partiel et diminue l'ambitus du spectre synthétique ${ }^{35}$.

Pour faciliter le repérage des différents éléments explicités dans les pages précédentes, nous avons volontairement ajouté des annotations et des zones surlignées au sein de la figure 11. Le motif de la contrebasse annonçant la sixième occurrence du spectre synthétique (première ellipse en gris clair) apparaît lors du transitoire d'extinction de l'occurrence précédente (voir l'occurrence « $5 »$ sur la figure 10). Au chiffre 6 de la partition est amorcée la sixième occurrence du spectre avec son transitoire d'attaque d'une durée de treize double croches, son régime stationnaire de trentesix double croches, et son transitoire d'extinction de treize double croches. La fondamentale $\left(m i_{1}\right)$ est restituée par le violoncelle, l'accordéon et la clarinette basse (la contrebasse est à l'octave inférieure). Les cordes et le cor restituent des composantes hors formant du spectre. Les composantes inclues dans les formants sont aisément repérables grâce aux indications $s$. dim. (sans diminuer).

Le formant principal est toujours constitué de deux groupes (rectangles en gris foncé), l'un bâti sur les harmoniques impairs (Prisme I) et confié aux instruments à vent dans la plupart des occurrences, l'autre bâti sur les harmoniques pairs (Prisme II) et confié exclusivement au percussionniste (glockenspiel puis vibraphone). Mais

35. Ce passage se situe entre 2'15' et 2'31' dans l'enregistrement de Partiels réalisé par l'ensemble Court-Circuit sous la direction de P.-A. Valade (CD G. Grisey, Les Espaces accoustiques, Accord Una Corda 465 386-2, 1999). Le spectre initial (voir fig. 4) est répété quatre fois (0'00"-1'05") avant de subir douze transformations successives (1'05'3'34'). 


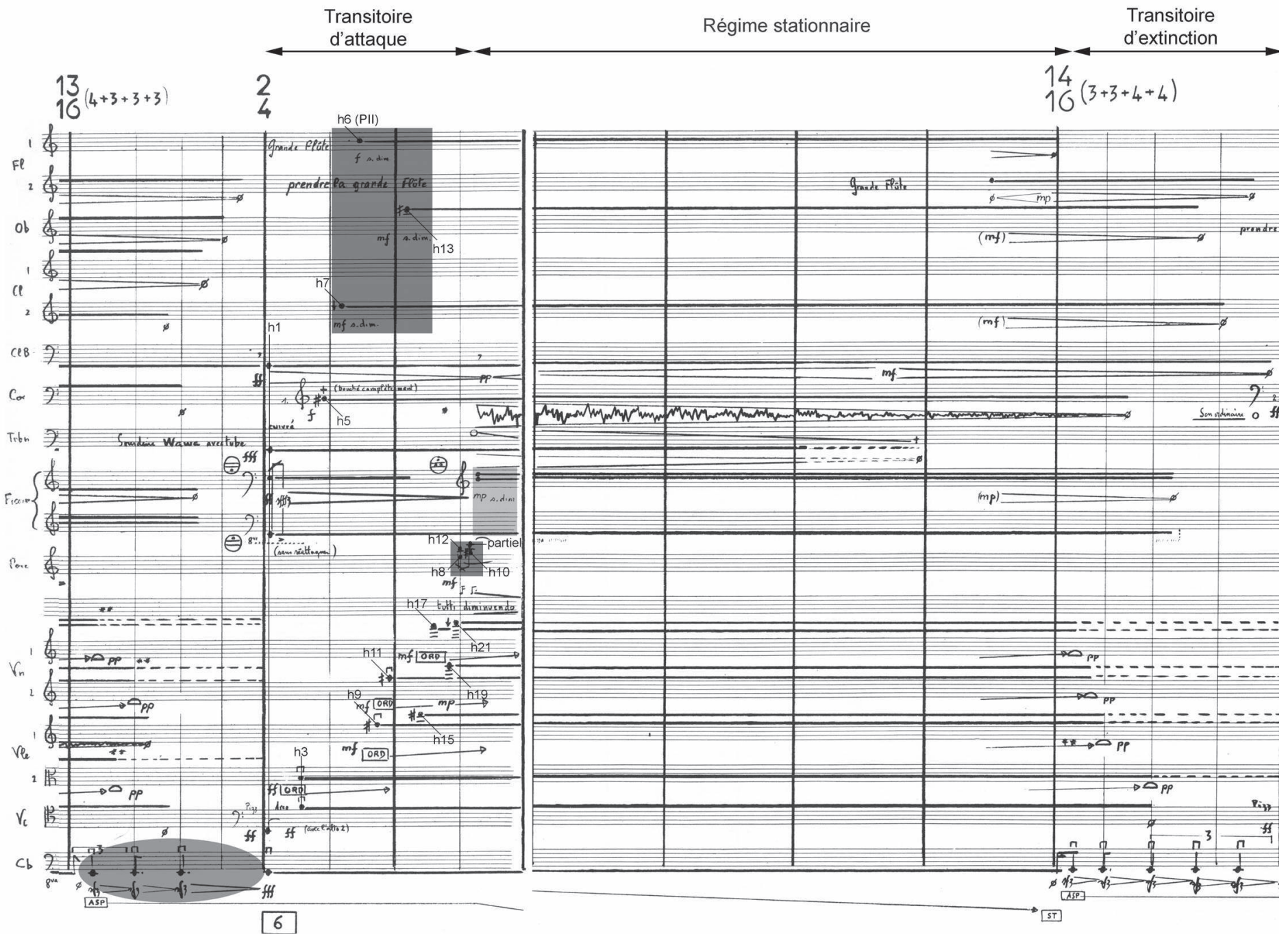

Fig. 11 : Partiels, p. 7 et 8 de la partition (Ricordi 132423, 1976). Cet extrait représente la fin de l'occurrence 5 du spectre synthétique et l'intégralité de l'occurrence 6 . Le motif rythmique de la contrebasse annonçant l'occurrence 6 est signalé par une ellipse en gris clair. Les différentes phases temporelles du spectre synthétique, ainsi que le numéro des harmoniques et la présence d'un partiel ont été rajoutés sur la partition. Le premier formant est signalé par deux rectangles gris foncé alors que le second formant (joué par l'accordéon) est signalé par un rectangle gris clair 
à partir de l'occurrence ici considérée, Grisey introduit systématiquement des notes étrangères à l'intérieur de ces deux groupes. Les harmoniques 7, 6 (note étrangère) et 13 , qui forment le premier groupe, sont confiés respectivement à la seconde clarinette, à la flûte et au hautbois. Les harmoniques $8,10,12$ et le $d o_{4}$ (note étrangère) sont joués par le percussionniste et constituent le second groupe. Cette intrusion d'un harmonique pair dans le premier groupe et d'un partiel dans le second groupe était parfaitement indiquée dans le plan structurel (voir l'indication « $1 \mathrm{P} \mathrm{II} \mathrm{+} 1$ partiel » sur la figure 8).

Le second formant (rectangle en gris clair), confié exclusivement à l'accordéon, clôture systématiquement la phase de restitution du transitoire d'attaque et annonce le début du régime stationnaire. Les notes restituées dans le cas présent $\left(m i_{1}, s i_{3}, r e_{4}\right)$ coïncident avec les harmoniques 1 , 6 et 7 (sans micro-intervalle). Elles ont été consciemment choisies par le compositeur pour renforcer les sons différentiels naturellement produits par la combinaison des trois notes jouées par la seconde clarinette (harmonique 7), la flûte (harmonique 6) et le hautbois (harmonique 13) : les différents couples d'harmoniques [13-6], [7-6], et [13-7] produisent respectivement, comme sons différentiels, les harmoniques 7, 1 et 6 (il suffit d'effectuer une simple soustraction).

Les quatre éléments transitoires notés par Grisey sur le plan synoptique (voir Fig. 8) correspondent au decrescendo de la clarinette basse (portée 12 en partant du bas), au crescendo-decrescendo qu'elle effectue ensuite, à l'instabilité du son au cor (signalé par un effet de gribouillage en dessous de la portée 12) et enfin, au relais entre les deux flûtes (portées 17 et 18). Les différences portant sur la structure interne du spectre synthétique n’ont pas été signalées car il faudrait visualiser l'occurrence précédente pour pouvoir les apprécier pleinement.

François-Xavier Féron est titulaire d'un master en acoustique musicale (Paris VI-Ircam) et d'un doctorat en musicologie (ParisSorbonne). Ses recherches se concentrent sur la dialectique Musique/Science. Après avoir enseigné à l'université de Nantes (20062008), il est parti à Montréal (McGill/CIRMMT) dans le cadre d'un contrat postdoctoral, puis a rejoint en 2009 l'équipe Analyse des pratiques musicales (Ircam-CNRS) pour mener une étude génétique sur Les Espaces acoustiques (1974-1985) de Gérard Grisey et Gramigna (2009-...) de Stefano Gervasoni.

François-Xavier Féron, feron@ircam.fr 